\title{
Forcing vine regrowth: a new technique to delay grape ripening until a cooler period
}

\begin{abstract}
>> One of the most important climate change-related effects on wine grapes is the advance in harvest period. A strategy to counteract this effect consists in postponing berry ripening until the conditions are cooler. A technique for forcing vine regrowth to delay grape ripening by more than two months was studied'. Forced vines produced smaller berries with lower $\mathrm{pH}$ and higher acidity and anthocyanin concentration than non-forced vines. $<<<$
\end{abstract}

\section{- The need for delaying berry ripening}

Global warming is an indisputable phenomenon. One of the most important climate change-related effects on wine grapes is the advanced time of harvest. Increased temperatures and warmer ripening periods can produce unbalanced wines with high alcohol levels, low acidities, modified varietal aroma and lack of color.

The latter is known to be a consequence of the decoupling effect of anthocyanin and sugar accumulation in red varieties ${ }^{2}$; that is, sugar accumulation in berries is accelerated, while phenolic maturity is retarded due to the inhibition of some related key enzymes in the metabolic pathways of phenol biosynthesis.

\section{- Available canopy management techniques}

One of the strategies for restoring the anthocyanin-tosugar ratio is to delay berry ripening until conditions become cooler $r^{3,4}$. To do this, several canopy management techniques have been proposed, such as late winter pruning, light or minimal pruning, apical leaf removal, application of antitranspirants and shoot trimming ${ }^{5}$. With each of these techniques, ripening can easily be delayed for 10 to 15 days, while a combination of several of them will potentially delay maturation for 15 to 45 days. This delay is long enough to postpone harvest until the onset of cooler weather conditions in most wine-growing areas and for most varieties.

However, there are extremely warm zones, such as the ones located in the $\mathrm{V}$ Winkler regions with more than 2,220 ${ }^{\circ} \mathrm{C}$ between April and October and where the earliest varieties, such as Chardonnay, are harvested in the first days of August (e.g., Montilla-Moriles and Ribera del Guadiana, Spain). In these cases, if the ripeness delay were approximately one and a half months, harvesting would take place in the middle of September, when average daily temperatures would still be quite high. We would therefore need to delay grape ripeness by two to three months, but in that case the above-mentioned techniques would not be enough.

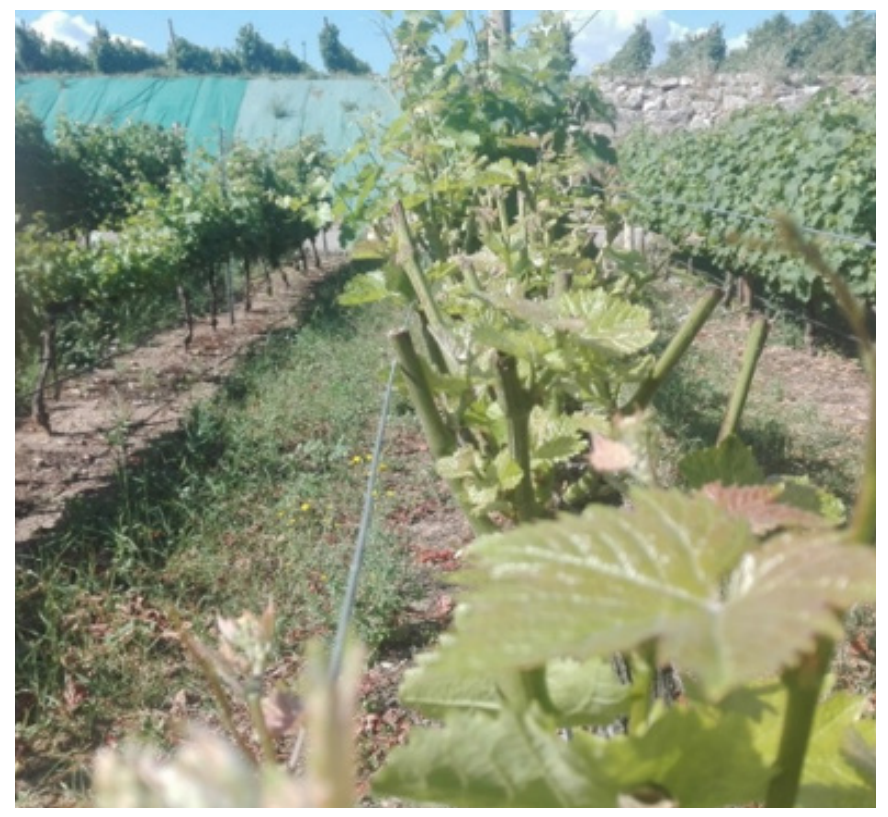

Figure 1. Forcing regrowth on shoots trimmed in late May.

\section{Technique for forcing vine regrowth}

With the aim of delaying grape maturation for at least two months, a technique based on forcing vine regrowth is proposed ${ }^{6,1}$.

This is a bold method for very hot viticultural regions which could be used to fight against climate warming. It consists in reducing growing shoots to several nodes with the aim of forcing vine re-growth; in order to force budbreak, shoot regrowth, and cropping, the source of inhibition needs to be eliminated and to this end, any existing lateral shoots, leaves and primary clusters are removed (Figure 1).

\section{- Delayed ripening}

When forcing was carried out at the stage in which inflorescences separated to bloom, fruit ripening shifted by more than one month, from a hot August to a cooler September/October period, which is better suited to producing quality grape for wine-making. Meanwhile, forcing after blooming shifted fruit ripening by more than two months, from a hot August to a cooler October/ November period.

\section{Chemical composition of the berry}

Forced vines produced smaller berries with lower $\mathrm{pH}$ and higher acidity and anthocyanins than non-forced vines.

Forcing is a promising way to restore the anthocyanin to sugar ratio decoupled by climate warming. 


\section{Yield reduction}

Forced shoots developed normally, but the number and weight of the clusters, as well as berry weight and yield, were generally lower than in the control, with high variability within the different years studied. Trimming to two nodes per shoot can also be applied as a good alternative to cluster thinning in order to reduce yield. Therefore, these results indicate that to achieve a yield comparable to the control more than two nodes per shoot should be left when trimming, if there is sufficient vigor for the development of more than two buds. More shoots per vine can also be left during the winter pruning. Future research on this technique should thus focus on improving yield.

\section{Conclusions}

Forcing regrowth effectively delayed all the phenological stages of grapevines to a great extent, causing ripening to occur at temperatures considerably lower than the control. Forced treatments reduced bunch number, bunch weight and vine yield. Early forcing treatments (when inflorescences separated) showed lower shoot fruiffulness than when postponed to the stages of bloom or post bloom. Therefore, in order to achieve a yield comparable to the control, more than two nodes per shoot should be left at trimming, if there is sufficient vigour for the development of more than two buds, and more shoots can also be left per vine during the winter pruning. Future research on this technique should thus focus on improving yield. Trimming to two nodes per shoot can be applied as a good alternative to cluster thinning. Forcing treatments can improve anthocyanin accumulation and help maintain a relatively high level of acidity in berries. Despite the risk of a serious decline in yield, forcing is a promising way to restore the anthocyanin to sugar ratio decoupled by climate warming. Further studies should be carried out to evaluate the long-term effects of forcing on grapevines. In addition, it would be interesting to apply this technique on different varieties and in other growing conditions to determine the optimum timing of forcing and the optimal number of nodes that should be left to obtain the desired yield.

\section{- Ideas for possible practical applications}

It should be first emphasised that this technique is only applicable when maturation needs to be delayed for well over a month; that is, in extremely hot areas. For most wine-growing regions of the world, such a long delay is not necessary; delays of less than a month are sufficient and can be achieved using other simpler techniques, such as late pruning, severe trimming or apical leaf removal.

Producers can test the forcing technique for themselves by cutting shoots during the flowering stage, leaving two to four nodes, and removing the leaves and any axillary shoots which have already emerged from the buds.
This technique does not pose any problems when pruning in the following year, since all shoots resulting from forcing are eliminated in the winter pruning. It should be borne in mind that for the following year the fertile shoots do not need to grow, but only shoots that can grow from the basal buds of primary shoots or on suitable secondary shoots, on which a new forcing treatment will be applied.

Although further studies are needed to evaluate the long-term effects of forcing on grapevines - especially on the nutritional reserves of perennial tissues - the results regarding the leaf area/yield ratio in the forcing treatments, which were much higher than $1.5 \mathrm{~m}^{2} \cdot \mathrm{kg}^{-1}$, seem adequate for maintaining reserves after severe canopy trimming, as well as for fast plant regrowth and very late-season ripening, bearing in mind that yields will be moderate. Such high leaf area/yield ratios indicate that vines possessed sufficient leaf area for their berries to mature properly and to accumulate reserves for the following years.

In situations for which this technique is applicable, its mechanisation is worth exploring: shoots could be cut mechanically on a horizontal plane, an operation which would be easier to perform in free shoot training systems than in vertical shoot positioning systems. Likewise, the post-cutting removal of leaves and any axillary shoots could also be done chemically; for example, by spraying a sufficient concentration potassium salts.

Fernando Martínez de Toda

Universidad de La Rioja/ICVV

Madre de Dios, 5126006 Logroño La Rioja

1 Martínez de Toda, F., García, J. and Balda, P. (2019) Preliminary results on forcing vine regrowth to delay ripening to a cooler period. Vitis, 58, 17-22. https://doi.org/10.5073/vitis.2019.58.17-22

2 Sadras, V. O. and Moran, M. A. (2012) Elevated temperature decouples anthocyanins and sugars in berries of Shiraz and Cabernet franc. Aust. J. Grape Wine Res. 18, 115-122.

3 Martínez de Toda, F. and Balda, P. (2013) Delaying berry ripening through manipulating leaf area to fruit ratio. Vitis 52 (4), 171-176. 4 Martínez de Toda, F., Sancha, J. C., Zheng, W. and Balda, P. (2014) Leaf area reduction by trimming, a growing technique to restore the anthocyanins:sugars ratio decoupled by warming climate. Vitis. 53 (4), 189-192.

5 Martínez de Toda, F. (2019) Técnicas vitícolas frente al cambio climático. Ed. Mundi-Prensa, Madrid, $175 \mathrm{pp}$.

6 Gu, S., Jacobs, S., McCarthy, B. and Gohil, H. (2012) Forcing vine regrowth and shifting fruit ripening in a warm region to enhance fruit quality in 'Cabernet Sauvignon' grapevine (Vitis vinifera L.). J. Hortic. Sci. Biotechnol. 87, 287-292. 